\title{
Emerging molecular mechanisms underlying cancer metastasis: the rising role of the long non-coding RNA GAS5
}

\author{
Marianna Lucafò ${ }^{1}$, Gabriele Stocco ${ }^{2}$, Giuliana Decorti ${ }^{1,3}$ \\ ${ }^{1}$ Department of Medicine, Surgery and Health Sciences, University of Trieste, Trieste, Italy; ${ }^{2}$ Department of Life Sciences, University of Trieste, \\ Trieste, Italy; ${ }^{3}$ Institute for Maternal and Child Health-IRCCS "Burlo Garofolo", Trieste, Italy \\ Correspondence to: Giuliana Decorti. Department of Medicine, Surgery and Health Sciences, University of Trieste, Trieste, Italy. Email: decorti@units.it. \\ Comment on: Chen L, Yang H, Xiao Y, et al. LncRNA GAS5 is a critical regulator of metastasis phenotype of melanoma cells and inhibits tumor \\ growth in vivo. Onco Targets Ther 2016;9:4075-87.
}

Submitted Sep 01, 2016. Accepted for publication Sep 07, 2016.

doi: $10.21037 /$ tcr.2016.10.01

View this article at: http://dx.doi.org/10.21037/tcr.2016.10.01

Metastasis is a multistep process, wherein cells in a primary tumor acquire invasive properties and disseminate throughout the body to establish secondary tumors at distant organs. During the metastatic cascade a complex interaction of signals are involved and identification of novel players in this process is crucial for cancer prognosis and for the development of agents effective on late stage malignancies. Although most of the studies in the literature focus on protein modulators of metastasis, the relevance of non-coding RNAs is now being appreciated. Long non-coding RNAs (lncRNAs) are commonly referred to as non-protein-coding RNA transcripts longer than 200 nucleotides. Emerging evidence has shown that lncRNAs are dysregulated in multiple cancer types and have a critical role in the process of metastasis (1). Of particular interest in this regard is the lncRNA GAS5 (growth arrestspecific transcript 5) which is down-regulated and inversely correlates with clinicopathological characteristics such as tumor size and metastasis progression in various human cancers, such as renal clear cells and bladder cancer $(2,3)$. However, studies on melanoma are still scarce.

Recently, Chen et al. (4) reported new insights on the role of GAS5 as regulator of metastasis phenotype in melanoma cells, testing the effects of modulating its expression by silencing and over-expression in in vitro melanoma cell models and in vivo in the nude mouse model. The authors demonstrated that GAS5 controls the metastasis phenotype via downregulation of the matrix metalloproteinases (MMPs) 2 and 9, whose activity has been implicated in proteolysis of the extra-cellular matrix (5). Of interest, previous studies have reported an association of MMP2 and
MMP9 expression with invasion and metastasis and poorer survival in different cancers, although with contrasting results (6). In primary and metastatic melanoma, MMP2 expression was significantly increased, was associated with worse survival of patients and was an independent molecular prognostic factor $(7,8)$.

Chen et al. (4) have shown that the expression of these metalloproteinases is inversely correlated with GAS5 levels: overexpression of GAS5 inhibits the expression of MMP2 and MMP9 whereas specific knockdown of GAS5 increased the proteinase expression (Figure 1A). However, the mechanism by which GAS5 regulates the transcription of MMP2 and MMP9 in vitro is largely unknown. In a previous paper (9), aiming to detect whether there is any direct interaction between GAS5 and MMP2, the same authors carried out an RNA pull-down assay, but the mass spectrometry could not demonstrate this kind of interaction. Given that a RNA-protein interaction has been excluded, it will be important to investigate, for example by chromatin immunoprecipitation, on the possibility that GAS5 is involved in regulating the transcription of MMP2.

GAS5 negatively regulates even the expression of vimentin, another protein associated with tumor invasion, in vitro and in vivo, as demonstrated by Chang and colleagues (10). In this paper, the expression level of GAS5 was examined in hepatocellular carcinoma and adjacent normal tissues of 50 patients, demonstrating that decreased expression of GAS5 was closely associated with the tumor differentiation. A significant negative correlation between GAS5 and vimentin levels was observed, again suggesting that GAS5 expression correlates with invasion processes. 
A

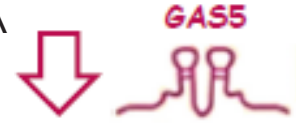

GAS5-AS1
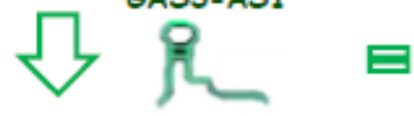

仓

VIMENTIN N-CADHERIN
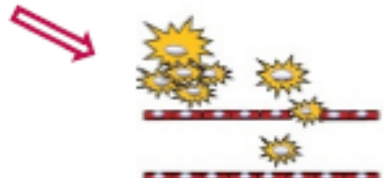

Metastasis

B

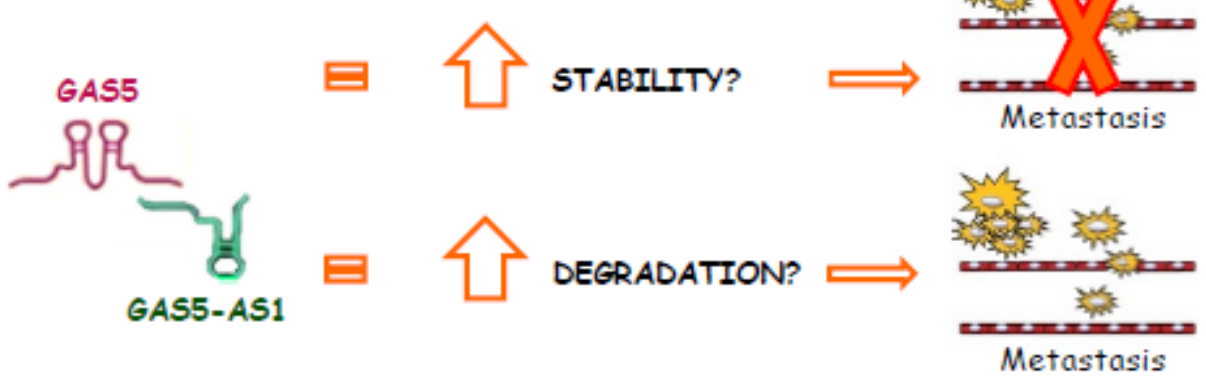

Figure 1 Putative regulatory role of GAS5 and GAS5-AS1 in metastasis phenotype. (A) Schematic illustration of the role of GAS5 and GAS5-AS1 in metastatic processes; (B) complementarity between the two lncRNAs could lead to an interaction that directly modulate RNA stability or degradation, providing an explanation for enhanced or diminished transcripts abundance, and consequent effect on metastatic process.

Relevant to this topic, Hu and colleagues (11) proposed GAS5 as endogenous "sponge" to regulate migration and invasion of hepatocellular carcinoma cells. In particular, GAS5 has a complementary region with miR-21, an important target of a large number of protein-coding transcripts that have a role in migration and invasion of cancer cells, and it can directly regulate miR-21 and its targets (11). Unfortunately, no data were reported about the role of GAS5 in the regulation of MMPs in hepatocellular carcinoma. Although Chen and colleagues (4) demonstrated that low expression of GAS 5 promotes invasion and migration by mediating MMP2 and MMP9 expression in in vitro cell models and in the nude mouse model, melanoma clinical samples are needed to confirm the molecular function of the lncRNA.

Consistent with these studies, recent investigation demonstrated that also the antisense RNA of GAS5, GAS5AS1 represses migration and invasion in non-small cell lung cancer cells without altering proliferation and survival (12). GAS5-AS1 is a lncRNA transcribed by a gene located in chromosome 1, as GAS5, and part of its sequence of nucleotides is complementary to the sense strand of GAS5. Reduced expression of GAS5-AS1 in non-small cell lung cancer samples as compared to the adjacent normal lung tissues was significantly correlated with tumor-nodemetastasis stage, tumor size, and lymph node metastasis while in vitro overexpression of GAS5-AS1 led to a dramatic inhibition of migration and invasion in different nonsmall cell lung cancer cell lines (12). To better understand the molecular basis responsible for the inhibition of migration and invasion mediated by GAS5-AS1, Wu and colleagues explored potential targets associated with cell migration and invasion. Elevated expression of GAS5AS1 in cells clearly decreased vimentin and several key epithelial-mesenchymal transition markers (Figure 1A). Intriguingly, GAS5 and GAS5-AS1 have a similar role in this context, although, at least in non-small cell lung cancer, they function in a different way: indeed, GAS5 mainly inhibits cell proliferation and induces apoptosis, while GAS5-AS1 represses cell migration and invasion. It will be interesting to study if the partial overlap of 40 or so 3' terminal nucleotides between the two transcripts has a role 
in the balance of the levels of the two lncRNAs and in the maintenance of these biological functions (Figure 1B).

Of particular interest is also the observation that some agents such as rapamycin have been proven to increase GAS5 expression (13) and this effect could contribute to their anti-tumor and anti-metastatic activity (14). Rapamycin has already been proposed as a co-treatment in some chemotherapeutic protocols, to increase the cytotoxic effect (15); given the mechanism highlighted in this commentary, this co-treatment strategy could improve the anti-metastatic effects, especially in patients with low levels of GAS5.

Careful and well-controlled investigations on the molecular mechanisms of lncRNAs activity are needed to understand their anti-metastatic and anti-proliferative effects; in particular, GAS5 and its antisense GAS5-AS1 seem particularly promising. These studies could provide new perspectives for lncRNA-directed diagnostics and antimetastatic drug targets.

\section{Acknowledgments}

Funding: None.

\section{Footnote}

Provenance and Peer Review: This article was commissioned and reviewed by the Section Editor Long Chen (Department of PET-CT Center at the Yunnan Tumor Hospital, The Third Affiliated Hospital of Kunming Medical University, Department of Biochemistry and Molecular Biology of Kunming Medical University, Kunming, China).

Conflicts of Interest: All authors have completed the ICMJE uniform disclosure form (available at http://dx.doi. org/10.21037/tcr.2016.10.01). The authors have no conflicts of interest to declare.

Ethical Statement: The authors are accountable for all aspects of the work in ensuring that questions related to the accuracy or integrity of any part of the work are appropriately investigated and resolved.

Open Access Statement: This is an Open Access article distributed in accordance with the Creative Commons Attribution-NonCommercial-NoDerivs 4.0 International License (CC BY-NC-ND 4.0), which permits the noncommercial replication and distribution of the article with the strict proviso that no changes or edits are made and the original work is properly cited (including links to both the formal publication through the relevant DOI and the license). See: https://creativecommons.org/licenses/by-nc-nd/4.0/.

\section{References}

1. Huarte $M$. The emerging role of lncRNAs in cancer. Nat Med 2015;21:1253-61.

2. Kong $\mathrm{H}, \mathrm{Wu} \mathrm{Y}, \mathrm{Zhu} \mathrm{M}$, et al. Long non-coding RNAs: novel prognostic biomarkers for liver metastases in patients with early stage colorectal cancer. Oncotarget 2016. [Epub ahead of print].

3. Ma C, Shi X, Zhu Q, et al. The growth arrest-specific transcript 5 (GAS5): a pivotal tumor suppressor long noncoding RNA in human cancers. Tumour Biol 2016;37:1437-44.

4. Chen L, Yang H, Xiao Y, et al. LncRNA GAS5 is a critical regulator of metastasis phenotype of melanoma cells and inhibits tumor growth in vivo. Onco Targets Ther 2016;9:4075-87.

5. Bauvois B. New facets of matrix metalloproteinases MMP2 and MMP-9 as cell surface transducers: outside-in signaling and relationship to tumor progression. Biochim Biophys Acta 2012;1825:29-36.

6. Tabouret E, Bertucci F, Pierga JY, et al. MMP2 and MMP9 serum levels are associated with favorable outcome in patients with inflammatory breast cancer treated with bevacizumab-based neoadjuvant chemotherapy in the BEVERLY-2 study. Oncotarget 2016;7:18531-40.

7. Poudel B, Lee YM, Kim DK. DDR2 inhibition reduces migration and invasion of murine metastatic melanoma cells by suppressing MMP2/9 expression through ERK/ NF- $\kappa B$ pathway. Acta Biochim Biophys Sin (Shanghai) 2015;47:292-8.

8. Rotte A, Martinka M, Li G. MMP2 expression is a prognostic marker for primary melanoma patients. Cell Oncol (Dordr) 2012;35:207-16.

9. Chen L, Yang H, Xiao Y, et al. Lentiviral-mediated overexpression of long non-coding RNA GAS5 reduces invasion by mediating MMP2 expression and activity in human melanoma cells. Int J Oncol 2016;48:1509-18.

10. Chang L, Li C, Lan T, et al. Decreased expression of long non-coding RNA GAS5 indicates a poor prognosis and promotes cell proliferation and invasion in hepatocellular carcinoma by regulating vimentin. Mol Med Rep 2016;13:1541-50.

11. Hu L, Ye H, Huang G, et al. Long noncoding RNA 
GAS5 suppresses the migration and invasion of hepatocellular carcinoma cells via miR-21. Tumour Biol 2016;37:2691-702.

12. Wu Y, Lyu H, Liu H, et al. Downregulation of the long noncoding RNA GAS5-AS1 contributes to tumor metastasis in non-small cell lung cancer. Sci Rep 2016;6:31093.

13. Mourtada-Maarabouni M, Hasan AM, Farzaneh F, et al. Inhibition of human T-cell proliferation by mammalian target of rapamycin (mTOR) antagonists requires

Cite this article as: Lucafò M, Stocco G, Decorti G. Emerging molecular mechanisms underlying cancer metastasis: the rising role of the long non-coding RNA GAS5. Transl Cancer Res 2016;5(Suppl 4):S827-S830. doi: 10.21037/tcr.2016.10.01 noncoding RNA growth-arrest-specific transcript 5 (GAS5). Mol Pharmacol 2010;78:19-28.

14. Yang Z, Lei Z, Li B, et al. Rapamycin inhibits lung metastasis of B16 melanoma cells through down-regulating alphav integrin expression and up-regulating apoptosis signaling. Cancer Sci 2010;101:494-500.

15. Niu H, Wang J, Li H, et al. Rapamycin potentiates cytotoxicity by docetaxel possibly through downregulation of Survivin in lung cancer cells. J Exp Clin Cancer Res 2011;30:28. 